Supporting Information for:

\title{
Reagent Triggers Isomerization of Fluxional Cluster Catalyst via Dynamic Coupling
}

Han Guo $^{[a]}$, Philippe Sautet ${ }^{*[a, b, c]}$, and Anastassia N. Alexandrova*[a,c]

[a] Department of Chemistry and Biochemistry, University of California, Los Angeles, Los Angeles, CA 90095-1569 (USA)

[b] Department of Chemical and Biomolecular Engineering, University of California, Los Angeles, Los Angeles, CA 90095-1569 (USA)

[c] California NanoSystems Institute, Los Angeles, CA 90095 (USA) 


\section{Theoretical Methods}

All electronic structure calculations are performed using the projector augmented-wave (PAW) potentials $^{1,2}$ and the Perdew-Burke-Ernzerhof (PBE) functional, ${ }^{3}$ implemented in the DFT-based Vienna ab initio simulation package $(\mathrm{VASP})^{1,4-7}$. The van der Waals interactions are treated with the $\mathrm{dDsC}$ dispersion correction. ${ }^{89}$ A $1 \times 1 \times 1$ Monkhorst-Pack $k$-point grid centered at $\Gamma$-point and a plane wave cutoff energy of $400 \mathrm{eV}$ are employed in all calculations, and the convergence criterion for the SCF relaxation is set to $10^{-6} \mathrm{eV}$. A large supercell of $16.6 \AA \times 16.4 \AA \times 16.7 \AA$ is used to ensure that the separation between repeated images is larger than $10 \AA$ under the periodic boundary conditions. The same SCF settings and supercell are used for transition state (TS) search and ab initio molecular dynamics (AIMD) calculations. TS search is performed using the climbing image nudged elastic band (CI-NEB) method, ${ }^{10,11}$ and the convergence criteria for residual forces are $0.01 \mathrm{eV} / \AA$.

We have performed AIMD calculations ${ }^{12}$ in an NVE ensemble to simulate $\mathrm{CH}_{4}$ impinging on the $\mathrm{Pt}_{13}$ cluster at a given temperature. The AIMD-QCT approach, which is widely used in gassurface dynamics studies, ${ }^{12-19}$ is a reasonable method to probe the reaction dynamics at a given substrate temperature when the total energy of the molecule is chosen well above the ZPEcorrected barrier, ${ }^{19,20}$ which is the case in our simulations. In this study, the lowest TS-energy sites on the global minimum of $\mathrm{Pt}_{13}$, reported in previous global optimization studies ${ }^{21,22}$, is chosen as the impact site, and this Pt atom is considered as the impact Pt for all the trajectories. To model the effect of cluster temperature, sixteen cluster equilibration runs of $1.5 \mathrm{ps}$ are initialized. The displacements from the global minimum and velocities of the Pt atoms are determined according to an independent harmonic oscillator model, in which the energy of each vibrational mode is sampled from a classical Boltzmann distribution. ${ }^{19,23}$ The initial conditions (velocities and 
positions) of the cluster in the production runs are randomly selected from the structures in the last $0.5 \mathrm{ps}$ of the sixteen equilibration trajectories. The configuration pool has an average temperature of $676.9 \mathrm{~K}$ with a standard deviation of $121.9 \mathrm{~K}$ for the target temperature of $700 \mathrm{~K}$, and the average temperature is $396.7 \mathrm{~K}$ with a standard deviation of $78.1 \mathrm{~K}$ for the target temperature of $400 \mathrm{~K}$.

For $\mathrm{CH}_{4}$, the vibrational ZPE is initially imparted to the molecule, and the initial vibrational states sample a Boltzmann distribution at an elevated temperature of $1500 \mathrm{~K}$ in order to observe more reaction events. ${ }^{15,19}$ Figure $\mathrm{S} 1$ shows the distribution of vibrational states for each vibrational mode. Note that we treat each vibrational mode independently. Thus for molecules with combination vibrations, we consider their contribution to multiple vibrational modes. Figure S1 shows that although the vibrational temperature is relatively high, most of the molecules are in the vibrational ground state for each vibrational mode, especially for those high-energy modes. For the low-energy triply-degenerated bending modes (mode v7, v8 and v9), $68 \sim 71 \%$ of the molecules are in the ground state for one mode, while $19 \sim 23 \%$ of the molecules have one quantum of energy for one mode, and $6 \sim 8 \%$ of the molecules have two quanta. Within these vibrational energy ranges, each vibrational mode can be treat as an independent harmonic oscillator. Note that we observed artificial intramolecular vibrational energy redistribution (IVR) caused by the resonant vibrations in methane molecule. However, we are not trying to compute the experimental reaction probability for molecules in certain vibrational state, but to observe the dynamic coupling between molecular reaction/collision and cluster isomerization. The initial rotational energy is set to zero, and the molecular orientation is randomly sampled. To minimize quantum effects such as tunneling,,$^{20,24,25}$ the incident translational energy is set to $0.19 \mathrm{eV}(1500$ $\mathrm{K})$, well above the dissociation barrier, and the methane molecule is initially placed at $4.5 \AA$ from 
the impact Pt atom, where the interaction between them is weak. The production trajectories are propagated with a time step of 0.5 fs for $1 \mathrm{ps}$. At the end of the simulations, we observed three outcomes: reaction, trapping and scattering. A molecule is considered reacted if one C-H bond reaches $2.5 \AA$ in length, and scattered if its center of mass reaches the distance of $4.0 \AA$ from the impact site. The remaining molecules are considered trapped. In order to compare cluster fluxionality in the presence of $\mathrm{CH}_{4}$ to that without $\mathrm{CH}_{4}$, we also performed reference AIMD calculations, in which $\mathrm{CH}_{4}$ is removed but the initial conditions of the cluster are kept the same as those in the production runs. 

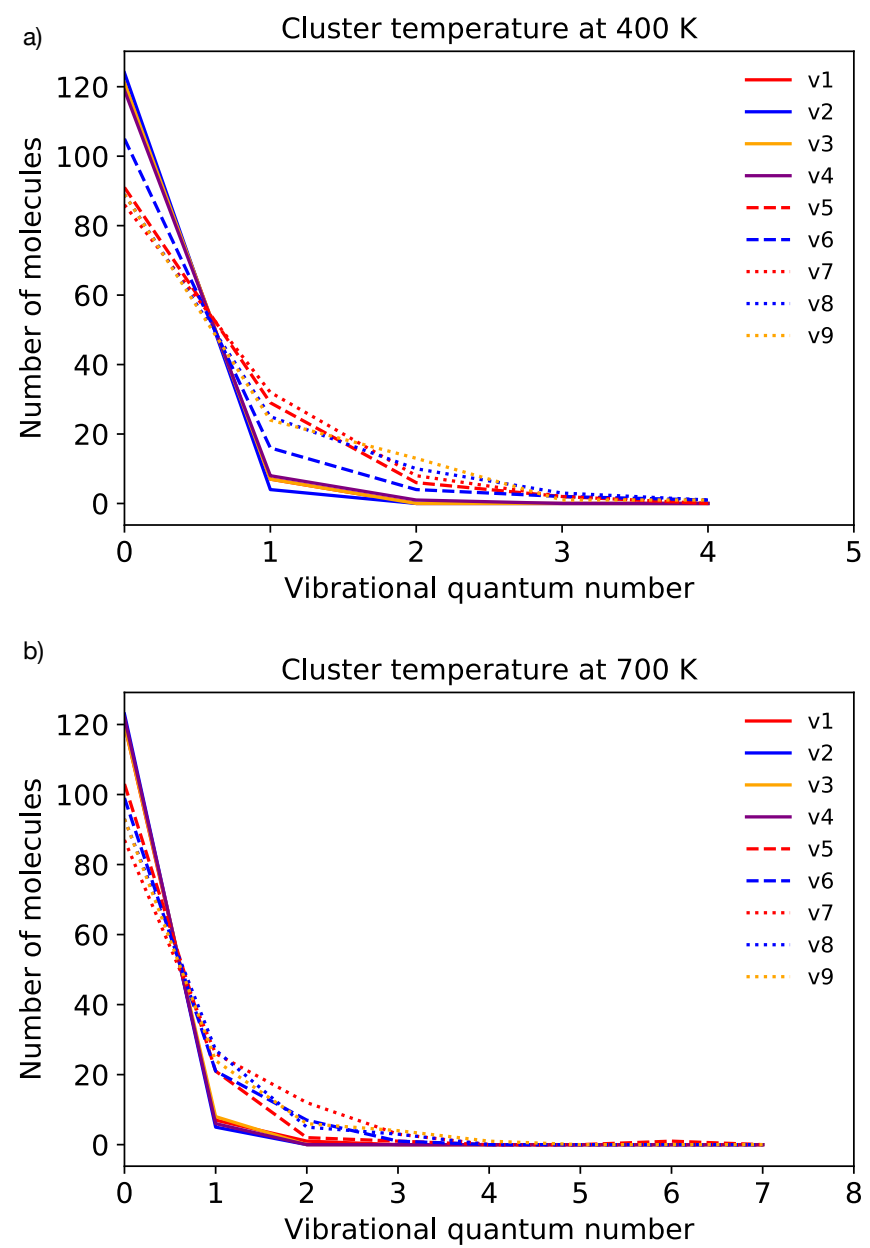

Figure S1. Distribution of vibrational quantum number for each vibrational mode. The results are shown for our simulations with cluster temperature of $400 \mathrm{~K}$ and $700 \mathrm{~K}$. 

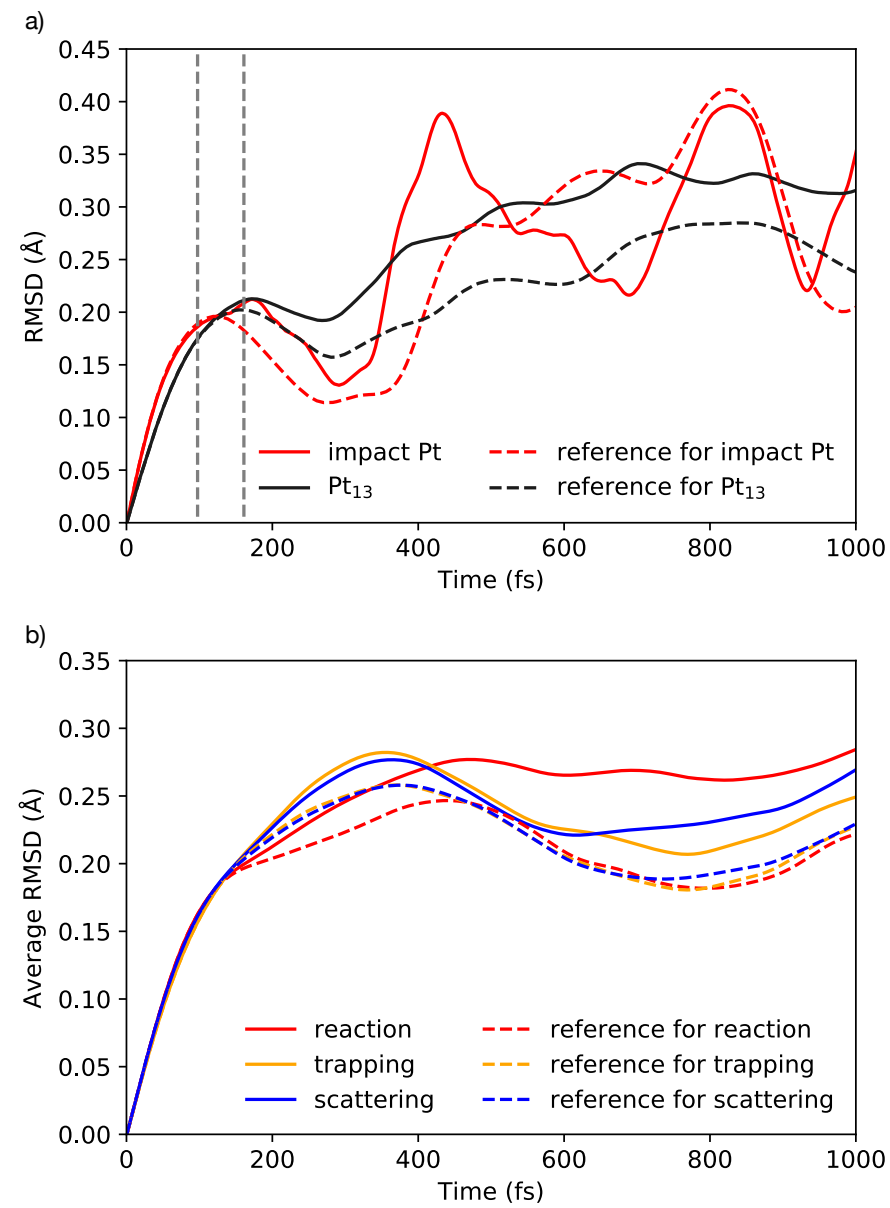

Figure S2. (a) RMSD of the impact $\mathrm{Pt}$ atom (red) and $\mathrm{Pt}_{13}$ (black) relative to the initial structure for one reaction trajectory and its reference trajectory at $400 \mathrm{~K}$. The first dashed grey line indicates methane arrival time (97.50 fs) and the second dashed grey line indicates the reaction starting time (161.00 fs). (b) Type-averaged RMSD relative to the initial structures for the production and reference trajectories at $400 \mathrm{~K}$. 

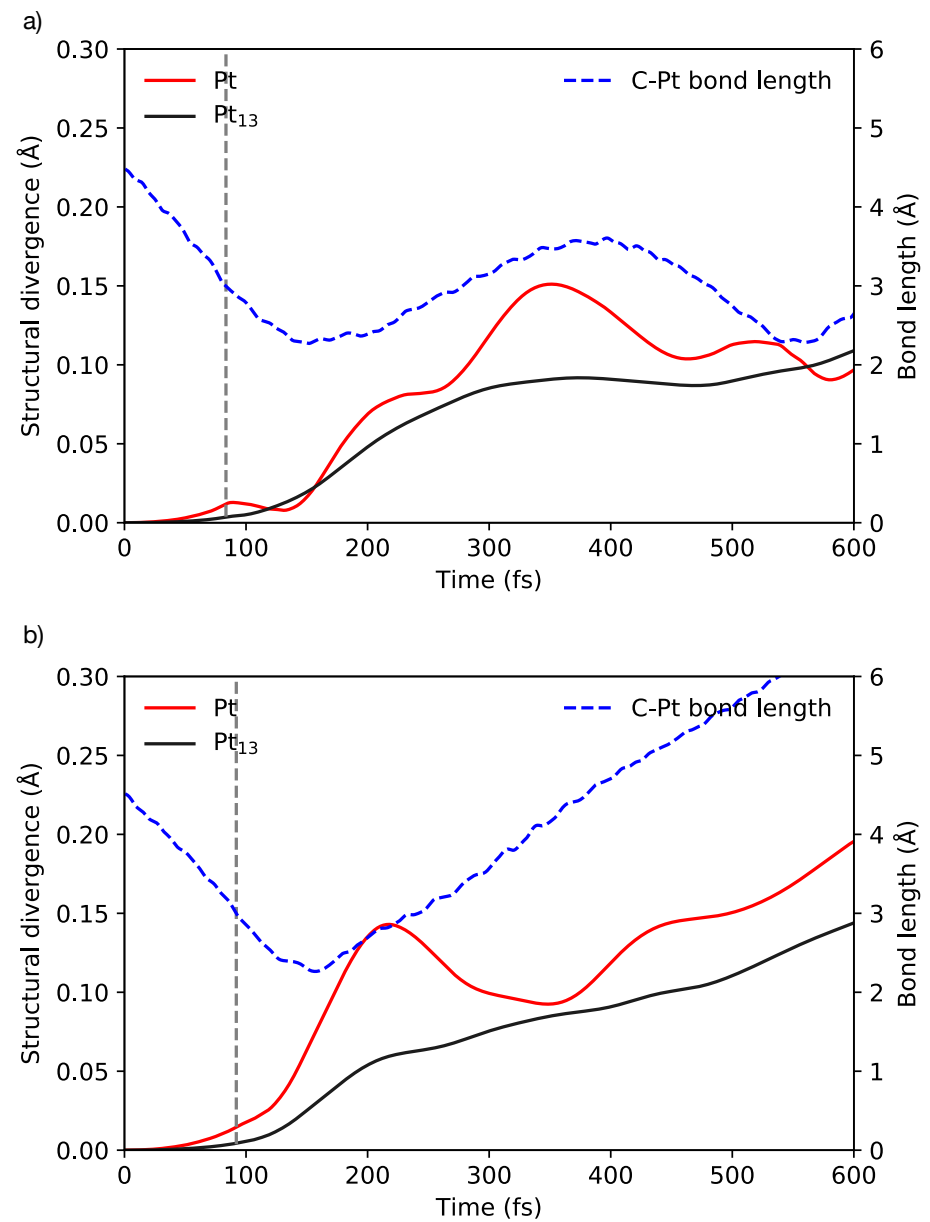

Figure S3. Structural divergence between one production trajectory and its reference trajectory for the impact Pt atom (red line) and $\mathrm{Pt}_{13}$ (black line) at $400 \mathrm{~K}$. The results are show for (a) trapping trajectory and (b) scattering trajectory. Pt-C distance is shown in blue dashed line. The vertical dashed line shows methane's arrival. 

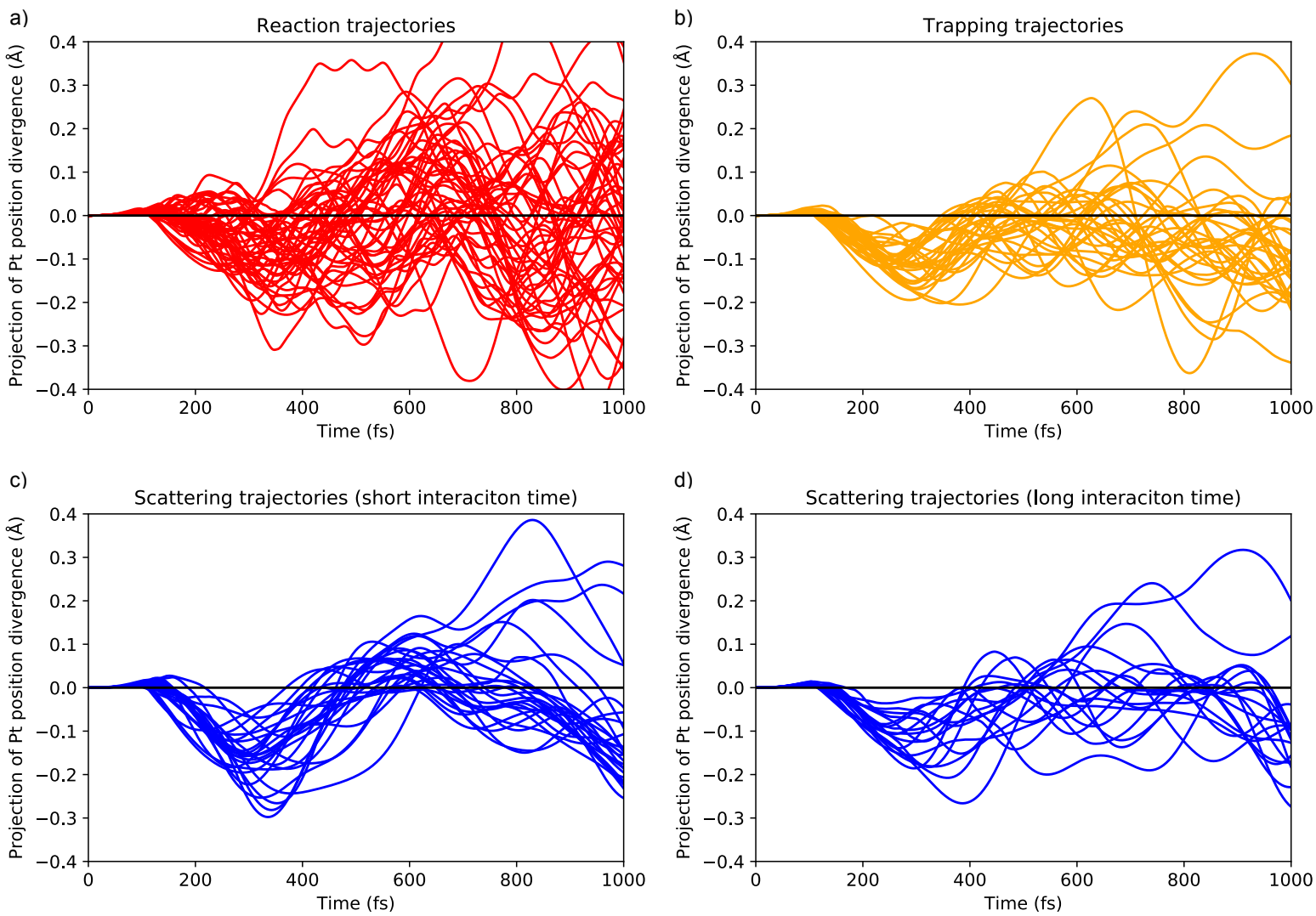

Figure S4. Projection of the position divergence of the impact Pt atom onto the direction along which $\mathrm{CH}_{4}$ comes in. Results are shown for four cases: (a) reaction (red), (b) strapping (orange), (c) immediately scattering (blue) and (d) scattering followed by trapping at $400 \mathrm{~K}$. 
a)

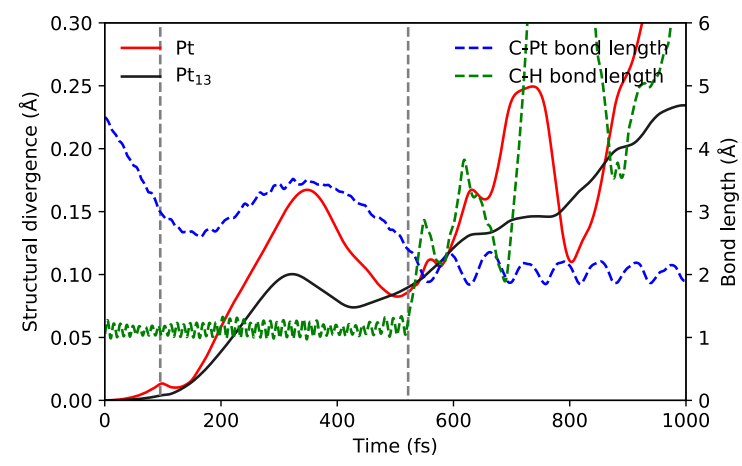

c)

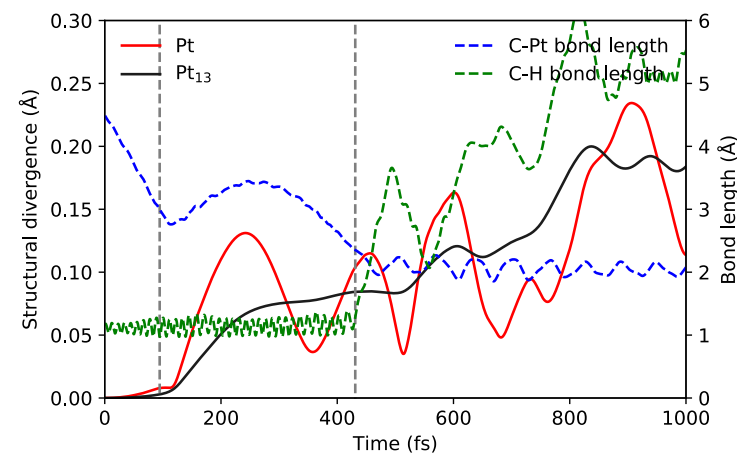

e)

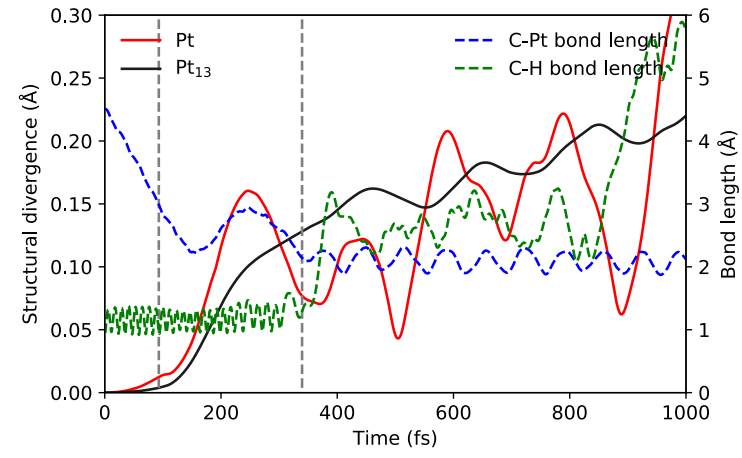

b)

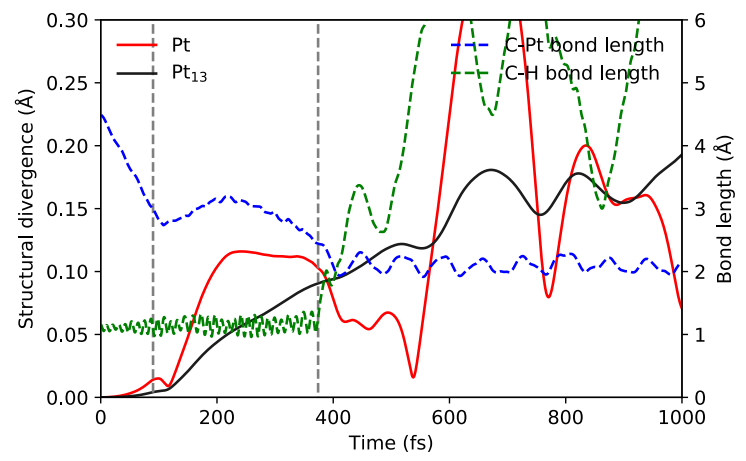

d)

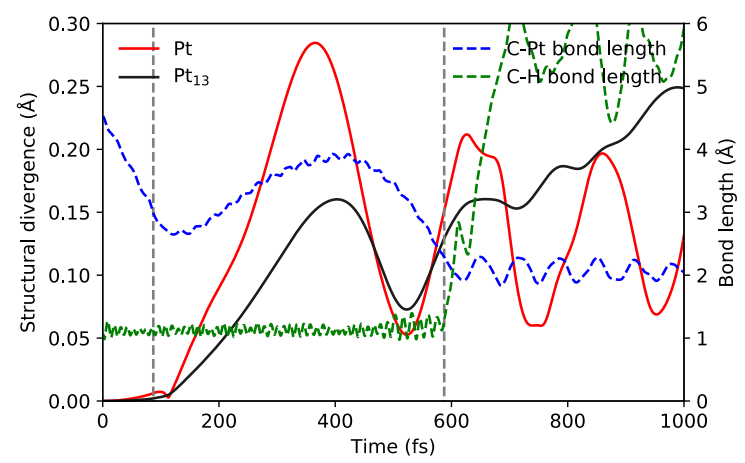

f)

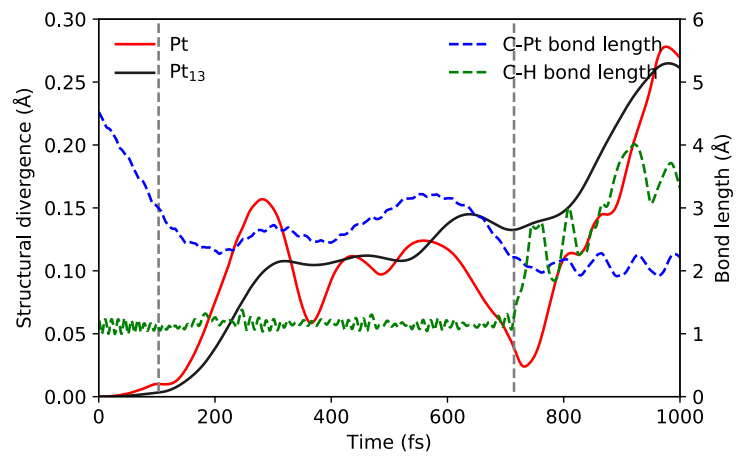

Figure S5. Structural divergence between production trajectories and their reference trajectories for the impact $\mathrm{Pt}$ atom (red line) and $\mathrm{Pt}_{13}$ (black line) at $400 \mathrm{~K}$. The results are shown for the 6 outliers in Figure 3(a). The dissociating $\mathrm{C}-\mathrm{H}$ bond length (green dashed line) 
and the Pt-C distance (blue dashed line) are shown in dashed lines. The first vertical dashed line shows methane's arrival time and the second vertical dashed line is the reaction starting time.

a)

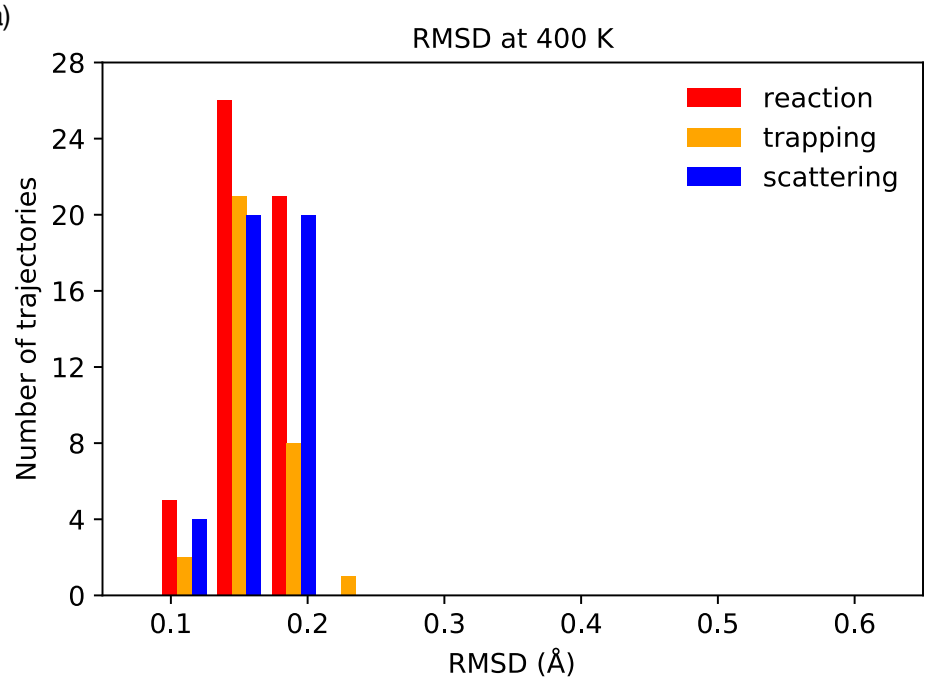

b)

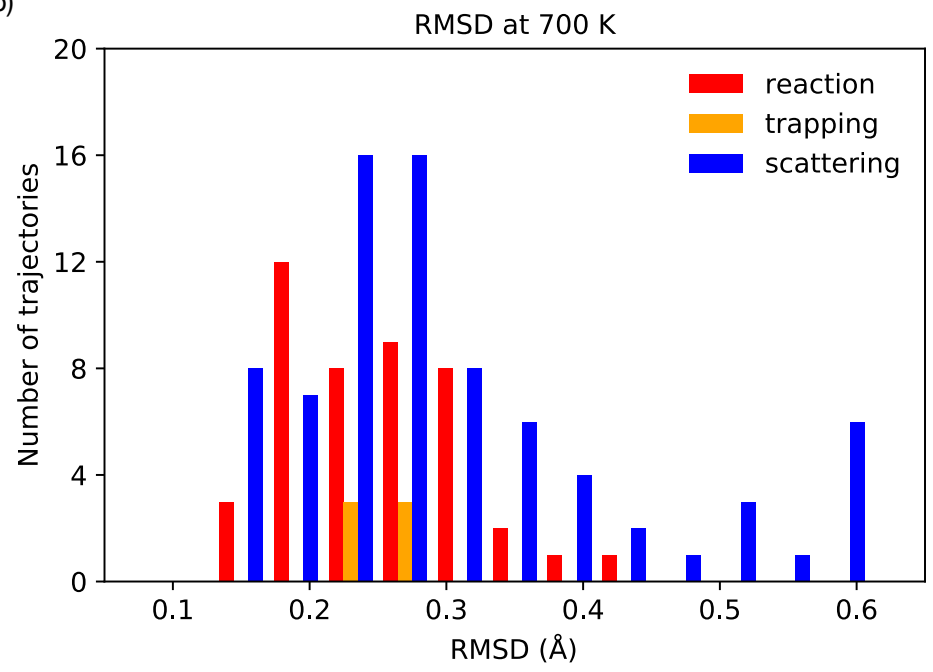

Figure S6. Distribution of RMSD relative to the equilibrium structure (global minimum) when $\mathrm{CH}_{4}$ arrives at the impact site at (a) $400 \mathrm{~K}$ and (b) $700 \mathrm{~K}$. 


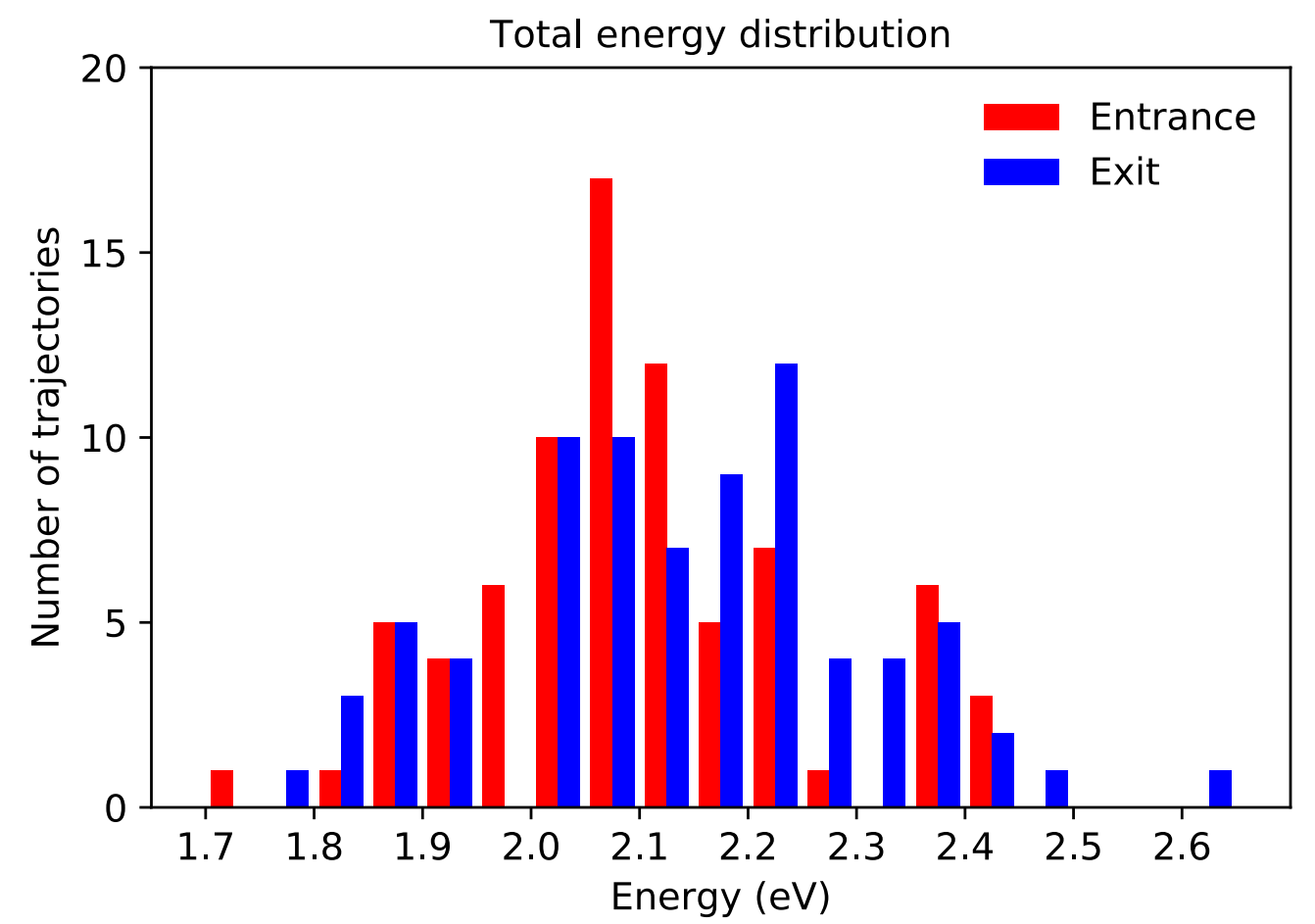

Figure S7. Cluster total energy distribution for all scattering trajectories at $700 \mathrm{~K}$. Results are shown for entrance channel ( $t=0 \mathrm{fs}$, red bars) and exit channel (methane leaves the cluster, blue bars) 
In order to analyze the effect of the adsorbates, we optimized the structures extracted from the exit channel of the reaction trajectories, without removing $\mathrm{CH}_{3}$ and $\mathrm{H}$. We divided the optimized structures into 3 groups at $400 \mathrm{~K}$ and 8 groups at $700 \mathrm{~K}$, based on which $\mathrm{Pt}_{13}$ isomer shown in Figure 4 will be obtained if the optimization is performed without adsorbates, and each group is called 'centered' at this $\mathrm{Pt}_{13}$ isomer. Figure $\mathrm{S} 8$ shows the energies for different groups of optimized $\mathrm{Pt}_{13}$ with adsorbates. For cluster temperature at $700 \mathrm{~K}$, we only plot the six lowest-energy groups, because for the two highest-energy groups the $\mathrm{Pt}_{13}$ configurations in the optimized structures with adsorbates are quite different from the $\mathrm{Pt}_{13}$ configurations obtained from optimization without adsorbates. 
a) $400 \mathrm{~K}$
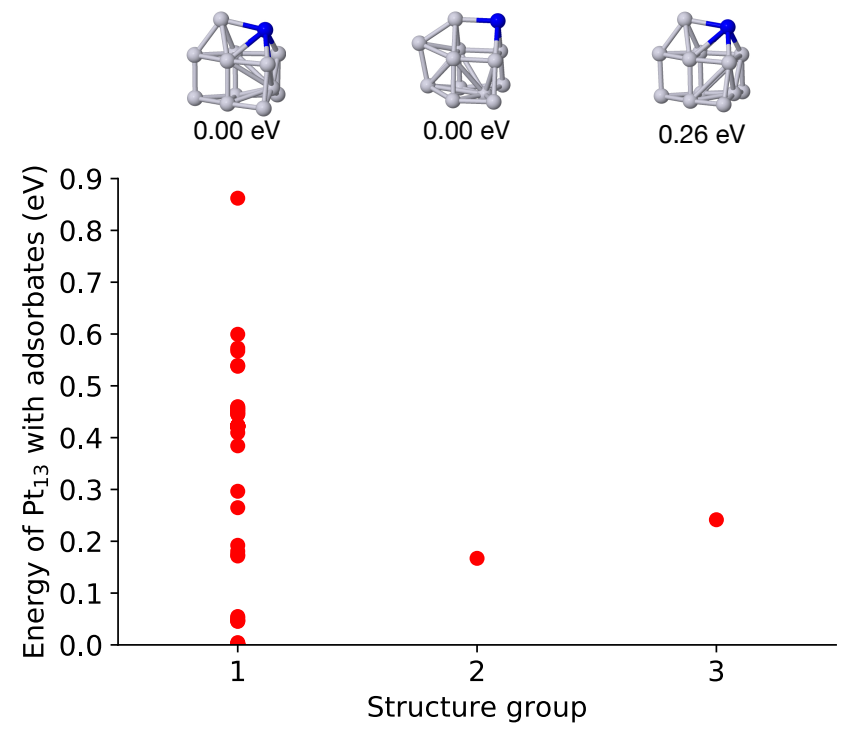

b) $700 \mathrm{~K}$

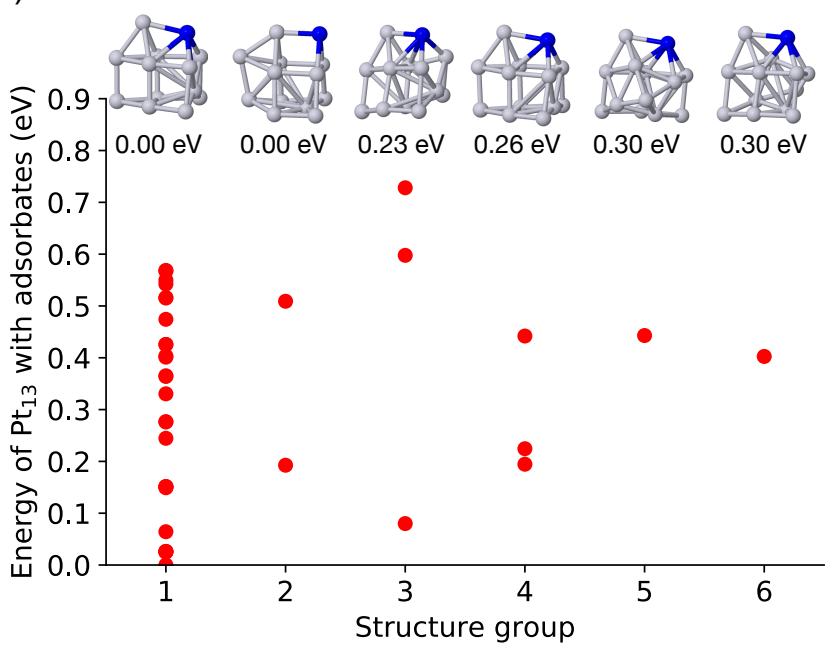

Figure S8. Relative energies for optimized $\mathrm{Pt}_{13}$ with adsorbates at $400 \mathrm{~K}$ (a) and $700 \mathrm{~K}$ (b). The structures are divided into different groups based on which $\mathrm{Pt}_{13}$ isomer shown in Figure 4 will be obtained if the optimization is performed without adsorbates. The $\mathrm{Pt}_{13}$ isomer and its relative energy is shown above each group. 
Table S1. Energy difference for different spin states.

\begin{tabular}{|l|l|l|}
\hline $2 \mathrm{~S}+1$ & $\Delta \mathrm{E}_{\text {vertical }}(\mathrm{eV})$ & $\Delta \mathrm{E}_{\text {adiabatic }}(\mathrm{eV})$ \\
\hline 1 & 0.141 & 0.140 \\
\hline 3 (global minimum) & 0.000 & 0.000 \\
\hline 5 & 0.190 & 0.190 \\
\hline 7 & 0.693 & 0.323 \\
\hline 9 & 1.202 & 0.657 \\
\hline
\end{tabular}

Reference

(1) Kresse, G.; Joubert, D. From Ultrasoft Pseudopotentials to the Projector AugmentedWave Method. Phys. Rev. B 1999, 59, 1758-1775.

(2) Blöchl, P. E. Projector Augmented-Wave Method. Phys. Rev. B 1994, 50, 17953-17979.

(3) Perdew, J. P.; Burke, K.; Ernzerhof, M. Generalized Gradient Approximation Made Simple. Phys. Rev. Lett. 1996, 77, 3865-3868.

(4) Kresse, G.; Furthmüller, J. Efficiency of Ab-Initio Total Energy Calculations for Metals and Semiconductors Using a Plane-Wave Basis Set. Comput. Mater. Sci. 1996, 6, 15-50.

(5) Kresse, G.; Furthmüller, J. Efficient Iterative Schemes for Ab Initio Total-Energy Calculations Using a Plane-Wave Basis Set. Phys. Rev. B Condens. Matter Mater. Phys. 1996, 54, 11169-11186.

(6) Kresse, G.; Hafner, J. Ab Initio Molecular-Dynamics Simulation of the Liquid-MetalAmorphous-Semiconductor Transition in Germanium. Phys. Rev. B Condens. Matter Mater. Phys. 1994, 49, 14251-14269.

(7) Kresse, G.; Hafner, J. Ab Initio Molecular Dynamics for Liquid Metals. Phys. Rev. B Condens. Matter Mater. Phys. 1993, 47, 558-561. 
(8) Steinmann, S. N.; Corminboeuf, C. A Generalized-Gradient Approximation Exchange Hole Model for Dispersion Coefficients. J. Chem. Phys. 2011, 134, 044117.

(9) Steinmann, S. N.; Corminboeuf, C. Comprehensive Benchmarking of a DensityDependent Dispersion Correction. J. Chem. Theory Comput. 2011, 7, 3567-3577.

(10) Henkelman, G.; Jónsson, H. Improved Tangent Estimate in the Nudged Elastic Band Method for Finding Minimum Energy Paths and Saddle Points. J. Chem. Phys. 2000, 113, 9978-9985.

(11) Henkelman, G.; Uberuaga, B. P.; Jónsson, H. Climbing Image Nudged Elastic Band Method for Finding Saddle Points and Minimum Energy Paths. J. Chem. Phys. 2000, 113, 9901-9904.

(12) Groß, A.; Dianat, A. Hydrogen Dissociation Dynamics on Precovered Pd Surfaces: Langmuir Is Still Right. Phys. Rev. Lett. 2007, 98, 206107.

(13) Füchsel, G.; Zhou, X.; Jiang, B.; Juaristi, J. I.; Alducin, M.; Guo, H.; Kroes, G. J. Reactive and Nonreactive Scattering of $\mathrm{HCl}$ from $\mathrm{Au}(111)$ : An Ab Initio Molecular Dynamics Study. J. Phys. Chem. C 2019, 123, 2287-2299.

(14) Zhou, L.; Jiang, B.; Alducin, M.; Guo, H. Communication: Fingerprints of Reaction Mechanisms in Product Distributions: Eley-Rideal-Type Reactions between D and CD 3 /Cu(111). J. Chem. Phys. 2018, 149, 031101.

(15) Migliorini, D.; Chadwick, H.; Nattino, F.; Gutiérrez-González, A.; Dombrowski, E.; High, E. A.; Guo, H.; Utz, A. L.; Jackson, B.; Beck, R. D.; et al. Surface Reaction Barriometry: Methane Dissociation on Flat and Stepped Transition-Metal Surfaces. J. Phys. Chem. Lett. 
2017, 8, 4177-4182.

(16) Zhou, X.; Kolb, B.; Luo, X.; Guo, H.; Jiang, B. Ab Initio Molecular Dynamics Study of Dissociative Chemisorption and Scattering of CO2 on Ni(100): Reactivity, Energy Transfer, Steering Dynamics, and Lattice Effects. J. Phys. Chem. C 2017, 121, 55945602.

(17) Jiang, B.; Yang, M.; Xie, D.; Guo, H. Quantum Dynamics of Polyatomic Dissociative Chemisorption on Transition Metal Surfaces: Mode Specificity and Bond Selectivity. Chem. Soc. Rev. 2016, 45, 3621-3640.

(18) Kolb, B.; Guo, H. Communication: Energy Transfer and Reaction Dynamics for DCl Scattering on Au(111): An Ab Initio Molecular Dynamics Study. J. Chem. Phys. 2016, $145,011102$.

(19) Nattino, F.; Ueta, H.; Chadwick, H.; Van Reijzen, M. E.; Beck, R. D.; Jackson, B.; Van Hemert, M. C.; Kroes, G. J. Ab Initio Molecular Dynamics Calculations versus QuantumState-Resolved Experiments on CHD3 + Pt(111): New Insights into a Prototypical GasSurface Reaction. J. Phys. Chem. Lett. 2014, 5, 1294-1299.

(20) Jiang, B.; Guo, H. Mode and Bond Selectivities in Methane Dissociative Chemisorption: Quasi-Classical Trajectory Studies on Twelve-Dimensional Potential Energy Surface. J. Phys. Chem. C 2013, 117, 16127-16135.

(21) Sun, G.; Sautet, P. Metastable Structures in Cluster Catalysis from First-Principles: Structural Ensemble in Reaction Conditions and Metastability Triggered Reactivity. $J$. Am. Chem. Soc. 2018, 140, 2812-2820. 
(22) Zhai, H.; Alexandrova, A. N. Fluxionality of Catalytic Clusters: When It Matters and How to Address It. ACS Catal. 2017, 7, 1905-1911.

(23) Nattino, F.; Díaz, C.; Jackson, B.; Kroes, G. J. Effect of Surface Motion on the Rotational Quadrupole Alignment Parameter of D2 Reacting on Cu(111). Phys. Rev. Lett. 2012, 108, 236104.

(24) Sacchi, M.; Wales, D. J.; Jenkins, S. J. Mode-Specificity and Transition State-Specific Energy Redistribution in the Chemisorption of $\mathrm{CH} 4$ on $\mathrm{Ni}\{100\}$. Phys. Chem. Chem. Phys. 2012, 14, 15879-15887.

(25) Sacchi, M.; Wales, D. J.; Jenkins, S. J. Mode-Specific Chemisorption of CH4 on Pt $\{110\}-$ (1x2) Explored by First-Principles Molecular Dynamics. J. Phys. Chem. C 2011, 115, 21832-21842. 